\title{
Le service domestique comme rapport social. Subjectivités au travail, emplois à domicile et migrations féminines en France
}

\author{
COLETTE LE PETITCORPS
}

Chez le docteur B. je faisais jeune fille au pair. Mais en fait quand on dit « jeune fille au pair », je faisais tout. Du moment qu'un jour elle est rentrée tard, je ne pouvais pas laisser les enfants, je me suis dit : «Il faut que je leur fasse à manger. » À partir de ce moment-là, j'ai tout fait. Mais tu crois qu'elle me payait plus? Je travaillais au noir à cette époque. Et puis j'ai rencontré mon mari, il m'a sortie de là, il m'a dit que c'était pas normal, que j'étais exploitée. Il m'a sortie de là, mais c'était pas mieux quand j'étais avec lui, je me suis retrouvée dans une autre situation plus difficile encore.

Cindy

Lorsque je rencontre Cindy en 2010, elle est à la retraite et depuis longtemps « sortie» du travail de «jeune fille au pair», expression pudique pour désigner la condition de bonne à tout faire. Elle relate ici son expérience passée comme employée de maison logée et nourrie alors qu'elle vient d'arriver en France : nous sommes dans les années 70, Cindy a 21 ans et elle a quitté l'Île Maurice pour fuir des contraintes familiales. À l'instar des 50 femmes migrantes de mon enquête de terrain, réalisée dans le contexte de ma thèse de doctorat, de 2011 à 2014 à Paris et à Poitiers (Le Petitcorps 2015), Cindy décrit l'exploitation vécue, de manière temporaire ou durable, dans le travail de service à l'intérieur de l'espace domestique d'autrui : à savoir un travail presque gratuit, sans limites de tâches ni de durée. Son récit met en scène la "patronne» en tant qu'agente centrale de l'exploitation de l'employée à domicile, dans un contexte d'emploi informel où la primo-arrivante est particulièrement vulnérable. Cindy mentionne aussi deux exploitations distinctes de son point de vue : celle du service domestique chez autrui et celle du service à son mari.

Alors que l'analyse féministe matérialiste inscrit l'exploitation du travail de service à domicile dans la continuité de celle du travail domestique gratuit des femmes, le discours de Cindy, comme celui d'autres migrantes employées à domicile enquêtées, mauriciennes, ivoiriennes et autres, semble vouloir dire autre chose. C'est à partir des expériences du travail de femmes migrantes, recueillies par des entretiens biographiques non directifs, longs et répétés, dans leur lieu de vie, que je cherche à comprendre la spécificité de l'exploitation qui a lieu dans le service domestique, dans ses liens aux processus migratoires féminins aujourd'hui. Or 
l'exploitation du service domestique constitue un problème théorique longtemps évacué de la littérature féministe en France (Fraisse 2009), que l'articulation des rapports sociaux de classe, de sexe et de race utilisée pour décrire les oppositions de classe et de race entre employeuses et employées domestiques de nos jours ne permet pas tout à fait d'élucider. J'argumente en effet que, en articulant ces rapports sociaux pour traiter de l'exploitation des femmes migrantes dans les emplois à domicile, les analyses intersectionnelles ou consubstantielles tendent à négliger l'application de certains principes des rapports sociaux qui permettent d'appréhender une société toujours en construction. L'articulation des rapports sociaux ne répond que partiellement à ce qui fait l'historicité du travail de service domestique en France et la matérialité de l'appropriation du travail de service, telle qu'elle est concrètement faite dans la relation de travail entre femmes. Les approches intersectionnelles tendent surtout à faire l'impasse sur la manière dont des groupes sociaux se produisent dans le rapport de service domestique. Or, les groupes sociaux qui se forment autour des enjeux du service domestique sont irréductibles aux catégories de race, de classe et de sexe par lesquelles la théorie de l'articulation des rapports sociaux tend à circonscrire les groupes dominants et dominés.

Je propose de conceptualiser un rapport social spécifique de service domestique, dans lequel les groupes de celles et ceux qui servent (le groupe servant) et de celles et ceux qui se font servir (le groupe servi) se produisent et s'affrontent, de manière divergente, face à l'enjeu du service domestique. J'entends montrer que la dynamique de ce rapport est aujourd'hui inextricablement liée aux processus migratoires féminins. Pour ce faire, je mobilise une démarche compréhensive des points de vue d'une cinquantaine d'employées à domicile migrantes (parmi lesquelles des militantes dans des syndicats), d'une vingtaine d'employeuses de services à domicile (de ménage, de garde d'enfants et d'aide aux personnes âgées), ainsi que des discours d'une quinzaine de responsables d'organismes intermédiaires de l'emploi à domicile'. À partir d'une relecture critique de la place et de l'analyse des domestiques dans la littérature féministe matérialiste, j'élaborerai d'abord le concept de rapport social de service domestique. Dans les trois parties suivantes, je confronterai ce concept aux données empiriques résultant de mon enquête de terrain sur les services à domicile et les migrations féminines en France.

\section{Le service domestique, le féminisme matérialiste et le rapport social}

Il faut noter d'entrée de jeu l'absence presque généralisée des domestiques dans les débats et la littérature féministes en France jusque dans les années $90^{2}$.

1 La méthode d'enquête que j'ai utilisée a principalement reposé sur le recueil de récits de vie et de discours par entretiens longs avec les personnes. Elle s'est accompagnée d'observations de relations entre des employeuses et des femmes de ménage à Paris.

2 Le même étonnant oubli pour une catégorie de main-d'oeuvre largement féminisée s'observe dans les féminismes ailleurs : en Amérique latine (Lautier 2002), en Europe 
LE SERVICE DOMESTIQUE COMME RAPPORT SOCIAL | 199

Attribuer cette omission au point de vue bourgeois des féministes du début du $\mathrm{XX}^{\mathrm{e}}$ siècle ou des matérialistes des années 70 serait trop simpliste. En effet, alors que l'unité de la bourgeoise et de l'ouvrière face au travail ménager apparaît bien dans les revendications féministes et les théorisations anciennes du travail domestique gratuit, la bonne, elle, en est exclue (Fraisse 2009). Qu'il y ait 700000 employées de maison déclarées par leurs employeuses ou employeurs dans les années 70 , sans compter le travail au noir (Fraisse 2009: 25), ne semble pas stimuler la critique féministe de l'époque. Dans son analyse de l'oppression des femmes, Colette Guillaumin (1978) mentionne l'existence de femmes payées pour accomplir une partie de la charge physique des membres invalides de la famille et des membres valides de sexe mâle. Cependant, elle leur consacre peu de lignes, en affirmant que ces tâches d'entretien physique sont, de toute façon, effectuées en grande majorité hors du circuit monétaire du travail (Guillaumin 1978). Le travail des domestiques est minoré par les approches féministes de l'exploitation du travail des femmes dans les années 70, et ce, d'un point de vue tant quantitatif que qualitatif. Ces approches n'analysent pas vraiment ce qu'apporte le fait de se faire servir par une personne étrangère à sa famille à la production sociale de l'existence, ni l'exploitation que ce travail de service contient. Le service domestique semble, en réalité, poser problème à la conceptualisation de l'exploitation des femmes par le travail domestique gratuit. En le prenant en considération, on veut non seulement rendre visible " un rapport direct d'oppression» entre femmes (Rollins 1990; Lautier 2002), mais aussi admettre que le mode de production domestique qui organise l'exploitation du travail gratuit des femmes puisse être affecté par cet autre travail domestique, payé.

De la marge, les employées à domicile apparaissent depuis les années 2000 au centre des analyses de l'exploitation du travail féminin dans la mondialisation néolibérale. Les migrations de femmes et l'apport de main-d'œuvre qu'elles représentent pour le secteur des services à domicile dans les pays du Nord retiennent aujourd'hui l'attention de travaux féministes, de la sociologie du travail et des migrations. En France, la politique des «emplois familiaux », puis des services à la personne, mise en place depuis les années 90 pour répondre au vieillissement de la population, à la généralisation de l'activité salariée continue des femmes et à la croissance du chômage, fait l'objet de plusieurs études. Celles-ci montrent que la politique publique choisie favorise des emplois féminisés, précaires, peu qualifiés, mal rémunérés et à temps partiel, aux dépens d'une intervention directe de l'État dans la prise en charge sociale des personnes vulnérables (enfants et personnes âgées notamment) (Devetter, Jany-Catrice et Ribault 2009; Dussuet 2002). Des travaux s'appuient sur la notion de division internationale du travail domestique pour mettre en évidence le rôle de l'État néolibéral et des politiques migratoires dans

(Anderson 2000) ou aux États-Unis (Glenn 2009). Les domestiques constituent en revanche l'une des figures centrales de l'exploitation des femmes noires aux États-Unis mise en exergue par le féminisme noir (Black feminism). 
l'exploitation du travail de migrantes qui contribuent en grande partie à la reproduction sociale au Nord (Scrinzi 2013; Falquet 2009). Le service domestique des femmes migrantes dans les foyers des cadres est analysé comme un travail devenu essentiel à la reproduction de l'organisation managériale du système capitaliste dans les villes globales ${ }^{3}$ (Sassen 2006). Des approches transnationales montrent comment le souci d'autrui (care), de femmes du Sud est extorqué pour les besoins de foyers du Nord (Hochschild 2004).

Les outils conceptuels du féminisme matérialiste incluent dorénavant employées et employés à domicile dans l'analyse de l'exploitation du « travail considéré comme féminin » (Falquet 2009). Les emplois domestiques deviennent ainsi exemplaires du processus d'inclusion des femmes dans le salariat selon des modalités sexuées que le concept de division sexuelle du travail entend décrire. Comme Francesca Scrinzi (2013: 60) le dit, ces emplois reposent sur «la dépendance et la disponibilité personnelle, des catégories mobilisées par les théories féministes pour analyser le travail domestique gratuit ». La notion d'externalisation du travail domestique, de son côté, traduit le phénomène d'intégration de tâches, auparavant exécutées gratuitement par les femmes dans la famille, au marché de l'emploi. Le service à domicile est donc, par ces approches, inscrit dans la continuité du travail domestique gratuit (Pfefferkorn 2012; Scrinzi 2013). Les emplois domestiques sont aussi pensés comme un exemple paradigmatique (Kergoat 2009) de l'articulation des rapports sociaux de sexe, de classe et de race. Ils consacreraient une opposition de classe "entre des femmes dont les capitaux économiques, culturels et sociaux vont en s'accroissant et des femmes, françaises et étrangères, de plus en plus nombreuses à se précariser et à s'appauvrir» (Kergoat 2009 : 122).

Les travaux qui mobilisent ces différentes notions du féminisme matérialiste passent sur l'exclusion première des domestiques du répertoire politique et conceptuel du féminisme. Or revenir sur l'existence de cette bévue dans l'histoire des idées féministes permet de remettre certains principes d'utilisation du rapport social au centre de l'analyse des emplois domestiques et des phénomènes migratoires qui lui sont liés. Prenons d'abord l'impératif d'historicité : admettre l'oubli des domestiques dans la littérature féministe passée conduit, de fait, à remettre en cause l'idée de «nouveauté » de la classe servile (Kergoat 2009; Falquet 2009). Il est donc question de replacer les services domestiques et la forme de leur exploitation dans une perspective historique. Cette approche conduit à resituer l'opposition de classe observée actuellement entre femmes, employées et employeuses de services à domicile, à sa juste échelle d'analyse et dans son temps. Ce qui se passe entre femmes dans l'espace domestique est de l'ordre d'une relation

3 Les villes globales désignent, selon la notion de Saskia Sassen (2006), les centres urbains qui concentrent les fonctions clés et les ressources de l'économie globale, tel que l'organisation et le management des grandes entreprises et des marchés financiers. Le développement de ces activités génère une demande de services qui sont mal rémunérés (femmes de ménage dans les bureaux, services domestiques du personnel, etc.). 
sociale entre des individus concrets, également distinguées par des rapports de race et d'extranéité/autochtonie aujourd'hui, qu'il convient d'inscrire dans un rapport plus abstrait ${ }^{4}$ et structurant sur le temps long, entre un groupe qui est servi et un groupe qui sert. Je nommerai ces deux groupes, le groupe servi et le groupe servant. Dans ce rapport se reproduisent les conditions objectives de servi et de domestique. Cependant, la naturalisation de sexe, de classe et de « race » du groupe servi et du groupe servant change au cours du temps, comme les travaux d'histoire sur la domesticité le montrent bien (Gutton 1981; Guiral et Thuillier 1991; Châtelain 1969; Sarti 2007).

Le principe de l'historicité permet de distinguer ce qui est de l'ordre des relations sociales entre femmes et ce qui a trait au rapport social de service, les deux formes ne convoquant pas les mêmes temporalités (Zarifian 2013). Il incite également à rediriger l'analyse sur la manière dont se fait concrètement l'appropriation du travail de service domestique pour l'usage privé des familles, en sortant d'une approche trop focalisée sur les appartenances sociales et les capitaux opposés des femmes en présence. Or cet impératif matérialiste nécessite, dans une analyse microsociologique fine de l'appropriation du travail, de remettre sur le tapis la relation d'oppression entre femmes. Il s'agit d'appliquer ici la démarche féministe de redéfinition extensive du travail, en ne caractérisant pas le service par les tâches effectuées, mais par le mode de production, le rapport et la hiérarchie sociale dans lesquels il s'accomplit. Dans cette logique, l'interaction entre l'employée et l'employeuse de services à domicile constitue bien une relation immanente à des individus concrets, mais qui se révèle particulièrement importante parce que le rapport social entre les groupes abstraits servi et servant s'y joue et s'y rejoue en permanence (Zarifian 2013).

Le problème qu'ont posé les domestiques au féminisme fait également ressurgir la tension entre femmes au cœur de l'analyse. L'absence de la voix des domestiques dans les travaux passés incite d'autant plus à explorer les pratiques sociales mouvantes et ambiguës des employées à domicile aujourd'hui. Je questionne leurs manières divergentes de « s'affronter à » (Zarifian 2013) l'enjeu du service domestique par rapport aux employeuses, mais aussi relativement à l'ensemble des personnes servies de même que des actrices et des acteurs du marché qui captent leur travail pour le bien-être et la reproduction des familles consommatrices de leurs services. J'examine ainsi les groupes qui se produisent dans les affrontements liés à l'enjeu du rapport social de service domestique.

La notion de «formes transitionnelles d'exploitation» de Guillaumin (1978 : 9) aide en partie à penser ce qui fait enjeu dans le service domestique. Elle permet en effet de décrire l'exploitation de tout travail qui se situe entre appropriation physique d'un groupe et accaparement de sa force de travail typique du régime salarial. La notion aide, de ce fait, à penser l'exploitation particulière des

4 Sur la distinction entre relation sociale et rapport social, voir Kergoat (2009). 
employées à domicile migrantes (Galerand 2015). J'envisage toutefois l'éventualité qu'elle ne puisse pas saisir tous les enjeux du service domestique, en raison de l'absence d'analyse de la contribution de ce travail à la production sociale de l'existence au départ. En revanche, Guillaumin souligne un aspect important de ce service lorsqu'elle désigne par le terme d' ' appropriées » les personnes qui sont les seules «à effectuer les tâches d'entretien matériel physique », dont les appartenances sociales de sexe, de race ou de caste varient selon les contextes: «Plus de $80 \%$ des personnels de service est composé de femmes en France, ces mêmes sont aux États-Unis des Afro-américains, femmes et hommes, en Inde des parias, hommes et femmes...» (Guillaumin 1978: 29). Si les personnes " appropriées » affectées aux tâches d'entretien matériel physique varient dans le temps et l'espace en fonction des hiérarchies sociales existantes, on peut oser penser d'abord un groupe des domestiques, ou des servantes et des serviteurs, sans a priori d'appartenance sociale : celui-ci se produit et se reproduit dans le procès de travail de service ainsi que dans le rapport au groupe servi, en tant qu'approprié. Il peut également devenir sujet politique. La question du processus de naturalisation du travail de service domestique à des femmes, des femmes migrantes et racialisées, vient ensuite, et en déplacer l'analyse permet d'envisager que les idéologies sexuées et racistes sur les qualités de celles et ceux qui effectuent ce travail bougent plus vite que le rapport personne servie/personne qui sert.

En partant de cette approche du service domestique par le concept de rapport social, je traiterai d'abord des conditions de travail des employées à domicile migrantes aujourd'hui en les situant dans le processus de reproduction historique des conditions de la bonne à tout faire. J'analyserai ensuite ce que constitue le travail de service domestique au regard de ce que représente le fait de se faire servir chez soi dans la matérialité du quotidien. Enfin, j'explorerai les pratiques communes ou collectivement organisées d'employées à domicile migrantes pour mettre en évidence les lignes de tension du rapport social de service domestique.

\section{Les migrations féminines et la reproduction de l'exploitation du service domestique}

Reprenons les propos de Cindy cités au début de mon article. Cindy dit qu'elle «fait tout» dans la maison de sa patronne, dans le contexte d'une relation d'emploi informelle et sans être payée de manière adéquate par rapport au temps de son travail de service. D'autres femmes migrantes de mon enquête m'ont fait part d'expériences analogues où elles exécutent un travail multiple qui s'étend jusqu'aux heures de la nuit, surtout lorsqu'elles sont logées et nourries chez la famille qu'elles servent. L'emploi sarcastique du terme de «bonne» rend compte dans leur récit de situations de travail où elles ont le sentiment de n'exister que par leur service pour la famille d'autrui. Quelles sont les conditions aujourd'hui réunies pour que des femmes éprouvent un statut de «bonne» dans les familles à un moment donné de 
LE SERVICE DOMESTIQUE COMME RAPPORT SOCIAL | 203

leur expérience de travail? J'examine des processus actuels de l'exploitation des employées à domicile migrantes qui reproduisent un rapport salarial doublé d'un lien de dépendance personnelle aux personnes servies, hérité de la domesticité.

Le premier processus d'entretien de cette exploitation particulière a trait au fait que la reproduction de la main-d'œuvre dans les emplois à domicile passe, pour une partie importante, par le renouvellement des migrations féminines et la venue constante de primo-arrivantes en situation vulnérable, du fait des politiques migratoires restrictives à l'immigration (Falquet et Moujoud 2010). Les parcours de femmes migrantes mauriciennes, qui deviennent employées à domicile - et sans papiers - à leur arrivée en France, montrent de manière exemplaire la façon dont les politiques migratoires et le fonctionnement spécifique du marché des emplois domestiques reproduisent la soumission, la surexploitation et la dépendance d'employées à domicile par rapport aux personnes qui les emploient.

Les femmes mauriciennes sans papiers arrivées en France durant les années 70, 80 et 90 (pour celles de mon enquête) trouvent le plus souvent un emploi à domicile par l'intermédiaire d'une compatriote avec un titre de séjour, qui est déclarée et réalise des heures de ménage auprès de sa clientèle fidélisée. Cette intermédiaire trouve un emploi à la primo-arrivante, qui est deux fois moins payé que le sien, non déclaré et souvent illimité en tâches et en temps de travail. Criminalisées par la politique migratoire, les travailleuses sans papiers se soumettent à ces conditions et se taisent par peur d'être arrêtées par la police. L'État joue alors un rôle dans l'entretien de leur exploitation en ne prévoyant pas de plan de régularisation de ces travailleuses, alors que les emplois à domicile sont bien identifiés parmi les secteurs d'activité nécessitant le recours à une main-d'œuvre étrangère (CAS 2006). L'État contribue ainsi à maintenir une partie du salariat en dehors de la protection juridique du travail, dans un secteur historiquement mis à l'écart du droit (Vasselin 2002). Les caractéristiques du marché des emplois à domicile sont aussi propices à la reproduction d'une situation de dépendance et de soumission de l'employée à ses employeuses ou employeurs analogue à ce que pouvaient vivre les femmes et les hommes qui quittaient la campagne pour s'engager dans la domesticité en ville au XIX ${ }^{\mathrm{e}}$ siècle (Châtelain 1969). Le fait que le domicile soit toujours un espace de travail soustrait de l'inspection du travail, que la rencontre de l'offre et de la demande soit encore principalement organisée de manière officieuse et que les employeuses ou les employeurs des classes possédantes se « recommandent » encore les employées à domicile, entraîne que les étrangères qui viennent d'arriver en terre inconnue sont à la merci des règles de ce marché organisé en grande partie hors des institutions classiques de l'emploi. Il se reproduit dans ces conditions ce qui constituait déjà la forme particulière du travail de service domestique au $\mathrm{XIX}^{\mathrm{e}}$ siècle, soit la condition domestique gagée, c'est-à-dire une dépendance à la famille servie assortie d'une rémunération (Gutton 1981) : «tu fais la bonniche, tu les suis partout, mais t'es payée quoi », comme l'énonce l'une des femmes ivoiriennes que j'ai rencontrées. 
Le second processus participe à l'entretien d'un rapport d'exploitation qui ressuscite l'idéal type de la «bonne à tout faire », dédiant sa personne tout entière à la vie sociale de la maisonnée (Simmel 1999). L'expansion des emplois à domicile de prise en charge des personnes dépendantes (enfants et personnes âgées) se révèle paradoxalement propice au retour d'un engagement subjectif et personnel de la travailleuse typique du domestique, auquel la femme de ménage embauchée pour des fonctions précises avait fini par échapper. Dans ces emplois de care, qui s'accompagnent depuis les années 2000 de la prolifération d'organismes privés de prestation de services à domicile, la finalité du travail est différente de celle de la femme de ménage, car il s'agit d'apporter « une réponse obligée à des besoins vitaux » (Damamme et Paperman 2009: 103). Cette finalité du travail se traduit par un souci de "professionnalisation» des métiers du care par les organismes intermédiaires de l'emploi, qui adoptent toutefois des pratiques et des discours contradictoires. Tout en recrutant des professionnelles diplômées, ces intermédiaires des emplois à domicile choisissent aussi des femmes non diplômées reconnues pour développer une sollicitude à l'égard des personnes fragiles sans compter les heures de travail. Cette disposition est attribuée à des caractéristiques raciales et culturelles qui font des «Africaines » les nounous ou les aides à domicile modèles sur le marché du care. Les catégories raciales de femmes migrantes sont cependant mouvantes. La directrice d'une association de gardes d'enfants à Paris affirme par exemple :

Par contre, il y a une nationalité qui est détestée partout sur Paris. Ce sont les Ivoiriennes. Elles ont un comportement très particulier, elles travaillent avec les enfants pour l'avantage financier, c'est tout. Et ce qui est incroyable aussi, c'est qu'elles sont arrivées en masse depuis quelques années chez nous, et qu'elles avaient toutes des CV et des lettres de motivations bien huilées, c'était trop beau pour être vrai. Vraiment on était ébahi par leur CV, mais aussi par la ressemblance entre les $\mathrm{CV}$ de chacune, à se demander si elles ne passaient pas par une association qui leur conseillait exactement ce que nous on recherchait.

Les femmes migrantes qui s'organisent entre compatriotes sur le marché des emplois à domicile pour trouver du travail, ou qui osent négocier leur salaire au premier entretien d'embauche, sont exclues de l'employée modèle africaine de care et écartées du recrutement par certains organismes. Les raisons pour lesquelles les Ivoiriennes sont éliminées de la catégorie raciale et culturelle de l'Africaine dévoilent l'outil de perpétuation de l'exploitation propre au service domestique que représente le processus de racialisation des qualités des travailleuses. La naturalisation des qualités de certaines femmes à délivrer du care par des organismes intermédiaires de l'emploi agit sur la forme du travail parce qu'elle tend à rétablir la norme du don de soi et de l'engagement entier de sa personne pour la vie de la 
LE SERVICE DOMESTIQUE COMME RAPPORT SOCIAL | 205

famille d'autrui. L'approche morale du souci d'autrui promue par ces organismes, en vertu de la finalité donnée au travail «nécessaire et vital» de care, paraît alors incompatible avec les revendications d'augmentation salariale des employées.

Le rapport d'exploitation dans le service domestique est étroitement lié de nos jours aux migrations féminines et à la façon dont celles-ci sont utilisées comme source de main-d'œuvre dans les emplois à domicile. Or ce qui affecte les femmes migrantes, que ce soit la condition sans papiers ou la racialisation de leurs qualités, conduit à assouplir l'ensemble du marché du travail visé (Marie 1984). L'inscription de ces processus d'exploitation au travail dans la continuité historique de la domesticité permet de faire ressortir l'enjeu du service domestique pour les personnes servies : il subsiste un intérêt à ce que le travail de service soit délivré dans des conditions qui perpétuent la dépendance des employées à ceux et celles qu'elles servent, pour le bien-être et la reproduction des familles de ces derniers. Que les employeuses ou employeurs soient « gentils » n'entrave pas la reproduction de ce rapport d'exploitation, parce que celle-ci est assurée par les acteurs du marché des emplois à domicile que sont les organismes intermédiaires de l'emploi et indéniablement l'État.

\section{Se faire servir : sur la nature du travail de service domestique}

Définir le service domestique comme un travail domestique payé est réducteur. Pour savoir ce qu'il constitue comme travail, il faut interroger ce qu'il apporte aux conditions matérielles de l'existence de celles et ceux qui sont servis à leur domicile par une personne étrangère à leur famille, et l'éventuelle hiérarchie sociale qui se reproduit dans sa réalisation concrète (Rollins 1990). Le concret et le quotidien du service s'organisent en règle générale dans la relation entre femmes, employeuses et employées, que ce soit pour le service ménager ou la garde d'enfants, dans une moindre mesure pour l'aide aux personnes âgées. Afin d'analyser les conditions matérielles de réalisation du service domestique, je m'appuie sur les expériences recueillies relativement au service de femmes migrantes qui travaillent comme garde d'enfants, femme de ménage ou aide aux personnes âgées au domicile d'autrui, d'une part, et sur les discours d'employeuses propriétaires de leur immobilier, avec leur conjoint ou seules, qui ont un diplôme d'études supérieures, d'autre part.

D'après l'expérience des employées à domicile migrantes de mon enquête, leur travail ne consiste pas simplement à se substituer à celui, gratuit, des femmes qui les emploient. Les employeuses tendent par exemple à ajouter, d'après les anecdotes relatées, du travail ménager qu'elles n'auraient sans doute pas effectué personnellement. L'exemple du tapis blanc que donne Bridget Anderson (2000) est éloquent: l'employeuse aurait-elle acheté un tapis de cette couleur, exigeant un nettoyage constant, si elle avait dû faire le ménage elle-même? La femme de ménage effectue bien souvent un travail en plus qui sert à la reproduction du style de vie des 
classes possédantes (Anderson 2000). Ce travail en plus, qui reste une éventualité banale et banalisée du quotidien des employées à domicile, matérialise de façon plus ou moins subtile une hiérarchie sociale qui se manifeste dans la relation concrète entre femmes. L'employée à domicile est parfois assignée à des tâches qui la renvoient directement à sa condition subalterne. Tout ce qui a trait au sale et aux produits du corps des personnes servies, comme un linge sale qui traîne et que l'employée doit ramasser, des toilettes souillées dans la cuvette et à l'extérieur de celle-ci, du sperme ou du sang laissés apparents sur le lit défait, ramène l'employée à domicile à son infériorité. Le rapport au sale d'autrui dans son intimité, que ce soit pour le nettoyer ou simplement le voir, ne renvoie pas tout à fait au même rapport que celui que l'épouse, la mère ou la fille a du produit des corps des membres de sa propre famille.

Le service domestique se caractérise de plus par une relation de travail qui suppose que les employées aient des "qualités passives », ce qui renvoie aux exigences envers les domestiques du XIX ${ }^{\mathrm{e}}$ siècle (Guiral et Thuillier 1991). La discrétion, l'adaptation, l'obéissance, une certaine façon de se présenter, de se tenir et de se vêtir, forment un savoir-être de l'employée attendu qui sert à maintenir chacune à sa place. En effet, l'un des soucis principaux des employeuses consiste à gérer l' « intruse dans la maison ». Or, s'assurer que l'employée ne dépasse pas son statut en marquant l'espace domestique de son empreinte, tout comme lui ajouter des tâches ou éventuellement établir ses bulletins de salaire, forment un ensemble de prérogatives féminines. Le rôle d'employeuse fait donc partie des tâches possibles du travail domestique gratuit. Cependant, la gestion d'une personne extérieure à la famille effectuant un service introduit une dimension supplémentaire à ce travail. L'employeuse est garante de l'entretien d'un ordre dans la maison où l'employée doit rester étrangère à la famille, même lorsque des relations intimes se nouent avec les autres membres de la famille (enfants, grand-père, etc.). Cela peut passer par un certain nombre d'injonctions et de rappels à l'ordre de l'employeuse à l'employée (comme ne pas changer l'emplacement des meubles qu'elle a choisi), voire par des sanctions. Dans plusieurs histoires recueillies, l'employée n'a pas été renvoyée parce qu'elle faisait mal le ménage, mais parce qu'elle était devenue trop envahissante dans l'espace domestique.

L'employeuse est l'intermédiaire par laquelle la propriété privée et l'intégrité de la famille sont entretenues malgré l'intervention d'une personne extérieure dans l'espace domestique. Son travail dans ce sens fait ressortir un enjeu de classe qui permet de comprendre la raison pour laquelle les besoins d'aide de certaines familles ne se traduisent pas automatiquement en recours au marché des services à domicile en France (Jany-Catrice 2016). Si la plupart des employeuses de l'enquête disent avoir employé quelqu'un lorsqu'elles ont commencé à travailler à l'extérieur de leur foyer ou quand elles ont eu des enfants, des entretiens approfondis révèlent que leurs parents employaient déjà une personne dans la maison lorsqu'elles étaient petites. Il pouvait s'agir d'une femme de ménage ou d'une bonne logeant à 
LE SERVICE DOMESTIQUE COMME RAPPORT SOCIAL | 207

demeure, travaillant aux côtés d'une mère au foyer, comme le raconte Geneviève, professeure des écoles à la retraite et mariée à un cadre :

Votre mère, elle, n'a jamais employé de femme de ménage...

$\mathrm{Si}$, d'ailleurs on a eu une bonne espagnole à un moment donné, c'était la mode, dans les années 60. Ma mère en avait besoin parce qu'on était sept enfants, donc c'était difficile à gérer, et déjà qu'elle était extrêmement nerveuse, à cause de sa fragilité aussi, j'imagine même pas si elle avait dû effectuer les tâches domestiques, donc c'était bien que de ce côté elle soit soulagée. En fait, ils ont pris cette bonne espagnole parce qu'à l'époque mon père travaillait dans une usine dans le XVII ${ }^{\mathrm{e}}$, et là-bas le personnel employait des bonnes espagnoles chez eux, donc ils ont dit à mon père qu'il fallait prendre une bonne espagnole, et donc mes parents ils ont écouté ce qu'on leur disait pour entrer dans le même monde bourgeois, c'était comme ça.

Les transmissions par les femmes de la condition de personne servie à son domicile d'une génération à l'autre sont le plus souvent niées dans les discours des employeuses. Bien que les usagères et les usagers de services n'aient pas toutes et tous d'expérience familiale du service domestique, on peut s'interroger sur ce que ces lignées existantes de familles servies, irréductibles à la haute bourgeoisie, produisent comme modèle de pratiques à imiter dans la manière de gérer et de considérer le travail de l'employée. L'analyse des expériences du service, d'employeuses et d'employées, fait apparaître un rapport de service différent de celui qui caractérise l'exploitation du travail de l'épouse. Le bénéfice du travail de service domestique, dans ce qu'il apporte aux conditions matérielles de l'existence, revient aux personnes servies qui incluent des hommes, des femmes et des enfants. Lorsque les employeuses, à l'instar de certaines analyses, considèrent le service d'employées comme un simple substitut du travail gratuit des femmes, elles reproduisent en réalité le même mécanisme qui entretient l'invisibilisation du travail domestique gratuit.

\section{Les tensions du rapport social de service domestique}

La stigmatisation des Ivoiriennes par des organismes intermédiaires de l'emploi dans l'exemple cité plus haut est un indicateur de tensions sur le marché des emplois à domicile. Les discours et les pratiques de structures intermédiaires de l'emploi cherchent à exclure de leur espace de contrôle du marché des services des femmes migrantes qui adoptent des pratiques de travail indésirables qui pourraient « contaminer» d'autres travailleuses. Cela amène à examiner quelles «lignes de tension» (Zarifian 2013) sont instillées par des pratiques communes ou collectivement organisées d'employées à domicile migrantes aujourd'hui, et le type de rapport de travail que celles-ci tirent du rapport de service domestique. 
Le premier enjeu de la lutte collective des femmes migrantes militantes enquêtées est d'agir sur la valeur salariale et la mesure temporelle de leur force de travail. Elles souhaitent ainsi accéder aux normes salariales du Code du travail, alors que le marché des emplois à domicile tend à entretenir la captation de leur travail, partiellement mesuré, et de leur personne pour le service à autrui. J'analyse ici les discours de deux militantes que j'ai rencontrées : une Mauricienne engagée dans la branche syndicale des employées de maison de la Confédération française démocratique du travail (CFDT) durant les années 80 et 90, et une femme ivoirienne qui préside un syndicat indépendant, spécialisé dans la défense des droits des "salariés du particulier employeur ${ }^{5}$ » et des auxiliaires parentales depuis les années 2010.

Hélène Capitaine, ancienne militante à la CFDT, luttait avec ses collègues mauriciennes employées de maison sans papiers au cours des années 80 et 90 pour la régularisation des travailleuses sans papiers. Le but de leur action politique était que les sans papiers soient incluses parmi les travailleuses salariées des emplois de maison et accèdent ainsi aux droits du travail garantis par la Convention collective nationale (la première convention pour les « employés de maison » datant de 1980). Cette convention garantit en effet une première mesure de la force de travail des employées et des employés à domicile: un taux de rémunération indexé sur le salaire minimum interprofessionnel de croissance (SMIC), le droit aux congés payés et aux congés maladie, la limitation légale du temps de travail, etc. La mobilisation du groupe des employées de maison sans papiers avait pour objet, dans le même temps, d'abolir le double marché des emplois à domicile qui entretient des niveaux d'exploitation différents :

Quand je suis arrivée ici, la seule chose que je ne voulais pas c'était me mettre en concurrence avec les travailleurs déjà installés ici, parce que d'abord il faut que je sache le prix d'une baguette de pain, lire la Convention collective des employées de maison, parce que sinon on sera deux clashs, et les employeurs ils vont bien profiter, ils aiment bien avoir une personne sans papiers, qui ne connaît pas ses droits pour l'exploiter. La seule façon, c'était de s'organiser.

(Hélène Capitaine)

Le discours de la présidente du syndicat des «salariés du particulier employeur » actuel montre en revanche que, même lorsque les employées à domicile migrantes sont régularisées et déclarées par leurs employeuses ou employeurs, elles doivent encore se battre pour faire respecter la Convention collective des salariés du particulier employeur ${ }^{6}$. En effet, dans ce travail, les personnes servies ne

5 Terme donné en France aux employeuses et employeurs de service à leur domicile.

6 Le texte actuellement en vigueur date de 1999. 
LE SERVICE DOMESTIQUE COMME RAPPORT SOCIAL |209

reconnaissent pas toujours aux travailleuses le droit d'être malades et d'être payées pour les heures de travail qu'elles font en plus du taux horaire convenu au moment du contrat. La mesure du temps de travail, pour être reconnues comme des travailleuses salariées, demeure donc centrale à la mobilisation d'employées à domicile migrantes, malgré le passage du temps et la signature de conventions. À noter que ce n'est pas propre au contexte français, puisque Elsa Galerand (2015) observe par exemple la même chose du militantisme des travailleuses domestiques philippines au Canada.

La mesure du temps de travail selon des normes salariales n'est toutefois pas la seule pratique politique que des employées à domicile migrantes adoptent pour changer les règles du travail de service domestique telles qu'elles sont établies sur ce marché. La théorie du sexage à laquelle se rapporte la notion de «formes transitionnelles d'exploitation » (Guillaumin 1978 : 9) fait défaut pour comprendre d'autres pratiques et revendications des travailleuses face à l'enjeu du service domestique. La nature spécifique de l'oppression des femmes selon le sexage est l'appropriation, de leur corps, alors que ce qui fait la classe des employées à domicile dans la lutte, ou des sans papiers parmi celles-ci, est le fait qu'elles sont à la fois «appropriées » et payées, sur un marché d'emploi en partie informel où les rémunérations et les temps de travail sont pluriels. Dans ce contexte, la mobilisation d'employées à domicile migrantes de même que les pratiques partagées par des migrantes qui ne sont pas engagées dans des syndicats, dépassent la question du respect du droit du travail selon la Convention collective. L'enjeu est en effet de transformer les dispositions dérogatoires au droit commun du travail, souvent pratiquées dans les relations de travail entre employeurs et employées, en occasion pour les employées à domicile de négocier le prix de leur service. Celui-ci se négocie au-dessus des taux de rémunération établis par la Convention collective (qui laisse peu de perspective d'évolution avec l'ancienneté), et des maigres salaires proposés par les organismes intermédiaires de l'emploi. Par exemple, bien que le fait de travailler au-delà de 48 heures par semaine en moyenne par trimestre soit contraire à la Convention collective, la présidente du syndicat indépendant avance que rien n'empêche les auxiliaires parentales de travailler plus, si du moins elles négocient une majoration des heures supplémentaires, elle aussi hors du cadre légal :

Travailler plus pour gagner plus, ça veut dire qu'au-delà de 40 heures les heures sont majorées à $25 \%$, au-delà de 48 heures elles sont majorées à $50 \%$, et moi je dis au-delà de 50 heures ils sont hors-la-loi, donc on peut négocier comme on veut, je dis les heures majorées à $100 \%$ ! À $100 \%$, puisque de toute façon on est hors du cadre légal.

(Militante ivoirienne)

Ces pratiques de militantes, et de non-militantes, ne consistent pas à faire glisser le rapport de service domestique d'un rapport d'appropriation à un rapport 
salarial, mais d'un rapport à la fois d'appropriation et salarial à un rapport où la dépendance de service est inversée et où le service devient un objet de commerce. La mesure de leur force de travail en argent, en temps de travail et en nature des tâches qu'elles introduisent devient alors plus précise et avantageuse que ce que prévoient la Convention collective et les organismes intermédiaires de l'emploi. Ces pratiques se comprennent en particulier au regard des parcours de migrantes qui insistent toutes sur le fait que les emplois à domicile constituent une solution matérielle afin de concrétiser des projets qui ont alimenté la décision d'émigrer. La rhétorique du combat caractérise les discours des femmes que j'ai rencontrées qui travaillent dans les emplois à domicile en vue de gagner de l'argent pour assurer, à leurs enfants et à elles-mêmes, un avenir autre que ce que la situation économique et sociale qu'elles ont quittée laissait présager. Il faut ajouter à cela que plusieurs femmes de mon enquête ont une expérience de travail passée comme commerçantes ambulantes. Elles disposent donc de capacités de négociation de leurs conditions de travail, si toutefois elles ne sont plus sans papiers et si elles se transmettent, entre collègues, des informations sur les « tarifs » pratiqués.

Les sujets politiques constitués dans les expériences syndicales enquêtées, d'employée de maison sans papiers ou d'employée à domicile migrante, ne contribuent pas uniquement à formuler des intérêts conflictuels par rapport à ceux des employeuses. En effet, la revendication de la régularisation administrative, en se constituant en sujet collectif employée de maison sans papiers par exemple, exprime aussi un rapport des travailleuses à l'État. L'enjeu du service domestique pour ces travailleuses consiste également à éliminer les formes de dépendance personnelle et d'appropriation de leur travail payé pour le service et le bien-être d'autrui. Or pour cela, l'action des militantes cherche à infléchir la façon dont fonctionne le marché des emplois à domicile et à y maîtriser la circulation des informations. Lorsque les militantes mauriciennes revendiquent la régularisation des employées de maison sans papiers, elles visent, au-delà de leur cas personnel, à supprimer le double marché des emplois à domicile où se reproduit une condition domestique en dehors de la protection du travail. De la même manière, le syndicat indépendant des « salariés du particulier employeur » actuel cherche à supprimer le système de la « recommandation » des employées entre employeuses, dans la perspective, à terme, de se constituer en agence de placement d'employées à domicile syndiquées. L'originalité des subjectivités politiques ne peut être saisie qu'en tenant compte des spécificités du marché des emplois à domicile, dans ce qu'il doit au passé de la domesticité et aux processus actuels de reproduction de l'exploitation du groupe qui sert au bénéfice de celui qui se fait servir.

\section{Conclusion}

L'analyse des emplois à domicile et des migrations féminines en France par le concept de rapport social de service domestique cible la production d'une 
LE SERVICE DOMESTIQUE COMME RAPPORT SOCIAL | 211

hiérarchie sociale, de conditions matérielles d'existence, de tensions et de subjectivités politiques dans le service domestique, plutôt que d'appréhender les relations de service à domicile entre femmes comme étant le seul produit d'autres rapports sociaux qui s'y imbriquent. La spécification d'un rapport social de service domestique a permis de saisir l'enjeu du travail de service pour le groupe servi. Pour celui-ci, l'enjeu consiste à conserver des conditions de service où se perpétue la dépendance des employées par rapport à celles ou ceux qui les emploient, ainsi que l'appropriation de leur personne pour le bien-être et l'entretien des familles servies, ce que les organismes intermédiaires de l'emploi et l'État concourent à préserver. L'affrontement des employées à domicile migrantes relativement à l'enjeu du service domestique, d'après les données de mon enquête, tente au contraire d'introduire une valeur financière et une mesure temporelle de leur force de travail qui suit les normes salariales et qui, de plus, déplace le rapport de service domestique, à la fois d'appropriation et salarial, vers un rapport où les employées négocient le prix de leur service au-delà des normes salariales. De leur maîtrise des règles et des informations circulant sur le marché des emplois à domicile dépend l'atteinte de cet objectif. Ces lignes de tension entre groupe servant et groupe servi se manifestent dans le contexte actuel d'une nouvelle géographie de la maind'œuvre de service avec le recours partiel aux femmes migrantes. Cependant, ces lignes de tension se comprennent aussi au regard d'une histoire longue de la domesticité qui continue de marquer la forme de ce marché de l'emploi, le type d'exploitation du travail et les pratiques tant des familles servies que des militantes.

Cette approche du service domestique par le rapport social invite à explorer les coproductions, davantage que les continuités, du service et du travail domestiques. Il s'avère essentiel en effet d'analyser la manière dont le rapport de service domestique et le rapport social de sexe constituent deux rapports de service différents, mais qui s'auto-entretiennent, en perpétuant la dévalorisation du travail de (re)production sociale des individus et des familles. L'analyse proposée pourrait également être utile pour examiner l'expansion d'autres formes d'exploitation des travailleuses et des travailleurs, qui émergent de la croissance des services et de l'effritement des rapports salariaux. En quoi l'organisation du travail de service domestique sert-elle de modèle à la transformation délétère des rapports de travail dans le système capitaliste mondialisé? Comment l'action politique d'employées à domicile migrantes, allant au-delà de revendications salariales, pourrait-elle inspirer les luttes dans d'autres secteurs d'activités, par l'intermédiaire de la condition migrante par exemple? Ce sont des questions que l'on peut dorénavant se poser.

\section{RÉFÉRENCES}

ANDERSON, Bridget

2000 Doing the Dirty Work? The Global Politics of Domestic Labour. Londres, Zed Books. 
CENTRE D'ANALYSE STRATÉGIQUE (CAS)

2006 «Besoins de main-d'œuvre et politique migratoire. Rapport au premier ministre », La Documentation française, [En ligne], [lesrapports.ladocumen tationfrancaise.fr/BRP/064000296/0000.pdf] (31 mai 2015).

CHÂTELAIN, Abel

1969 "Migrations et domesticité féminine urbaine en France, XVIII $-\mathrm{XX}^{\mathrm{e}}$ siècle », Revue d'histoire économique et sociale, 47, 4 : 506-528.

DAMAMME, Aurélie, et Patricia PAPERMAN

2009 «Care domestique: des histoires sans début, sans milieu et sans fin», Multitudes, 37-38: 98-105.

DEVETTER, François-Xavier, Florence JANY-CATRICE et Thierry RIBAULT

2009 Les services à la personne. Paris, Éditions La Découverte.

DUSSUET, Annie

2002 «Le genre de l'emploi de proximité », Lien social et Politiques, 47 : 143154.

FALQUET, Jules

2009 «La règle du jeu. Repenser la co-formation des rapports sociaux de sexe, de classe et de "race" dans la mondialisation néolibérale », dans Elsa Dorlin (dir.), Sexe, race, classe, pour une épistémologie de la domination. Paris, Presses universitaires de France : 71-90.

FALQUET, Jules, et Nasima MOUJOUD

2010 «Cent ans de sollicitude en France », Agone, $43: 169-195$.

FRAISSE, Geneviève

2009 Service ou servitude : essai sur les femmes toutes mains. Lormont, Le Bord de l'eau éditions.

GALERAND, Elsa

2015 «Quelle conceptualisation de l'exploitation pour quelle critique intersectionnelle? », Recherches féministes, 28, 2 : 179-197.

GLENN, Evelyn Nakano

2009 «De la servitude au travail de service : les continuités historiques de la division raciale du travail reproductif payé », dans Elsa Dorlin (dir.), Sexe, race, classe, pour une épistémologie de la domination. Paris, Presses universitaires de France : 21-70.

GUILLAUMIN, Colette

1978 «Pratique du pouvoir et idée de Nature (1). L'appropriation des femmes », Questions féministes, $2: 5-30$.

GUIRAL, Pierre, et Guy THUILLIER

1991 La vie quotidienne des domestiques en France au XIX ${ }^{e}$. Paris, Hachette Littérature.

GUTTON, Jean-Pierre

1981 Domestiques et serviteurs dans la France de l'ancien régime. Paris, Aubier. 
HOCHSCHILD, Arlie Russell

2004 «Le nouvel or du monde », Nouvelles Questions féministes, 23, 3 : 59-74.

JANY-CATRICE, Florence

2016 «Les “services à la personne" en France. L’impasse de stratégies univoques de croissance économique », Revue française des affaires sociales, $1: 263-278$.

KERGOAT, Danièle

2009 « Dynamique et consubstantialité des rapports sociaux », dans Elsa Dorlin (dir.), Sexe, race, classe, pour une épistémologie de la domination. Paris, Presses universitaires de France : 111-125.

LAUTIER, Bruno

2002 «es employées domestiques latino-américaines et la sociologie : tentative d'interprétation d'une bévue », Cahiers du genre, 32 : 137-160.

LE PETITCORPS, Colette

2015 Service à domicile, femmes et migrations en France. Le rapport de domesticité en question. Thèse de doctorat (sociologie). Poitiers, Université de Poitiers.

MARIE, Claude-Valentin

1984 « De la clandestinité à l'insertion professionnelle régulière. Le devenir des travailleurs étrangers régularisés ", Travail et emploi, $22: 21-31$.

PFEFFERKORN, Roland

2012 Genre et rapports sociaux de sexe. Lausanne, Éditions Page deux.

ROLLINS, Judith

1990 «Entre femmes. Les domestiques et leurs patronnes », Actes de la recherche en sciences sociales, 84, $1: 63-77$.

SARTI, Raffaela

2007 «Variations sur le thème de la dépendance. Femmes et domestiques entre Ancien Régime et Modernité », Les Cahiers du Centre de recherche historique, 40 : 99-109.

SASSEN, Saskia

2006 «Vers une analyse alternative de la mondialisation : les circuits de survie et leurs acteurs », Cahiers du genre, 40, $1: 67-89$.

SCRINZI, Francesca

2013 Genre, migrations et emplois domestiques en France et en Italie. Construction de la non-qualification et de l'altérité ethnique. Paris, Éditions PETRA.

SIMMEL, Georg

1999 «Excursus sur la frontière sociale », dans Georg Simmel (dir.), Sociologie. Études sur les formes de la socialisation. Paris, Presses universitaires de France : 609-612. 
LE PETITCORPS | 214

VASSELIN, Karine

2002 «Faire le ménage: de la condition domestique à la revendication d'une professionnalité », dans Françoise Piotet (dir.), La révolution des métiers. Paris, Presses universitaires de France : 77-98.

ZARIFIAN, Philippe

2013 « Rapport social de service, client et valeur», La Nouvelle Revue du travail, 2, [En ligne], [nrt.revues.org/737] (6 septembre 2015). 